\title{
Design of Ultrasonically-Activatable Nanoparticles using Low Boiling Point Perfluorocarbons
}

\author{
Paul S. Sheeran ${ }^{1}$, Samantha Luois ${ }^{2,3}$, Lee Mullin ${ }^{1}$, Terry O. Matsunaga ${ }^{3}$, and Paul A. \\ Dayton $^{1}$ \\ ${ }^{1}$ Joint Department of Biomedical Engineering, The University of North Carolina and North \\ Carolina State University, 304 Taylor Hall, CB 7575, Chapel Hill, NC 27599, USA \\ 2Undergraduate Biology Research Program, University of Arizona, PO Box 245067, Tucson, AZ \\ 85724, USA \\ ${ }^{3}$ Department of Radiology Research, University of Arizona, PO Box 245067, Tucson, AZ 85724, \\ USA
}

\begin{abstract}
Recently, an interest has developed in designing in biomaterials for medical ultrasonics that can provide the acoustic activity of microbubbles, but with improved stability in vivo and a smaller size distribution for extravascular interrogation. One proposed alternative is the phase-change contrast agent. Phase-change contrast agents (PCCAs) consist of perfluorocarbons (PFCs) that are initially in liquid form, but can then be vaporized with acoustic energy. Crucial parameters for PCCAs include their sensitivity to acoustic energy, their size distribution, and their stability, and this manuscript provides insight into the custom design of PCCAs for balancing these parameters. Specifically, the relationship between size, thermal stability and sensitivity to ultrasound as a function of PFC boiling point and ambient temperature is illustrated. Emulsion stability and sensitivity can be 'tuned' by mixing PFCs in the gaseous state prior to condensation. Novel observations illustrate that stable droplets can be generated from PFCs with extremely low boiling points, such as octafluoropropane (b.p. $-36.7^{\circ} \mathrm{C}$ ), which can be vaporized with acoustic parameters lower than previously observed. Results demonstrate the potential for low boiling point PFCs as a useful new class of compounds for activatable agents, which can be tailored to the desired application.
\end{abstract}

\section{Keywords}

ultrasound; nanoparticle; fluorocarbon; phospholipid; thermally responsive material; acoustically responsive material

\section{Introduction}

The ability to selectively trigger changes in the physical properties of a material or compound has led to a number of innovations in biomedical technology. Many activatable

(C) 2012 Elsevier Ltd. All rights reserved.

Correspondence to: Paul A. Dayton.

Publisher's Disclaimer: This is a PDF file of an unedited manuscript that has been accepted for publication. As a service to our customers we are providing this early version of the manuscript. The manuscript will undergo copyediting, typesetting, and review of the resulting proof before it is published in its final citable form. Please note that during the production process errors may be discovered which could affect the content, and all legal disclaimers that apply to the journal pertain. 
'intelligent materials' and nanoparticles have been designed to respond to external stimuli such as electric/magnetic fields, heat, light, and sound [1]. In the field of ultrasound medical imaging, recent interest has focused upon the development of phase-change contrast agents (PCCAs), which convert from a liquid to a gaseous state upon exposure to thermal and/or acoustic energy [2]. Many of the unique applications proposed for PCCAs take advantage of the volumetric increase that occurs during vaporization. Microscale droplets limited to flow in the vascular space have been proposed for a number of techniques, including temporary occlusion of blood vessels and enhancing cavitation activity [3-6]. PCCAs manufactured with diameters in the 100-nm range may be able to diffuse out of the characteristically 'leaky' vasculature of many solid tumors before phase-transition [7], providing a means of contrast-enhanced extravascular imaging, cell-specific targeting, drug delivery, and therapy via ultrasound $[8,9,10]$.

Currently, the only ultrasound contrast agents (UCAs) approved by the FDA for clinical applications consist of lipid-coated microbubbles with gaseous perfluorocarbon (PFC) cores [11]. The high molecular-weight gaseous PFC core is favored due to its low solubility, which provides enhanced circulation lifetime. The significant difference in density and compressibility between the gas-core and surrounding fluid makes these microbubbles highly echogenic and readily detectable with an ultrasound imaging system [12, 13]. In addition, the perfluorocarbon dose used for typical clinical and pre-clinical studies results in few to no adverse bioeffects [14]. Though they are restricted to vascular flow due to size, their potential use for echocardiography, vascular perfusion imaging, drug/gene delivery, and enhancement of cavitation/thermal-based therapies has been studied extensively [1523]. Several groups have investigated alternative ultrasound contrast agents based on inert liquid perfluorocarbon cores, and although the contrast provided is inherently less than that of UCAs, they have some echogenicity due to a higher density and a lower speed-of-sound than water and tissue [24, 25]. Like their gas-core counterparts, these emulsions are nontoxic in small doses, but can be designed to have greater stability in circulation than typical UCAs $[13,26]$.

Phase-change contrast agents can be considered a hybrid of the gas-core UCAs and the inert liquid core droplets in that the perfluorocarbons selected have a boiling point near enough to body or room temperature that they can be 'tipped' from the liquid phase to the highly echogenic gas phase through the addition of thermal and/or acoustic energy. The result is a dynamic, high-contrast agent with very low toxicity that can be activated with spatial and temporal specificity $[2,27,28]$. Additionally, through the naturally high fluorine content of PFCs or through incorporation of nanoparticles/fluorescent dyes, use of these agents can be extended to other imaging modalities such as ${ }^{19} \mathrm{~F}$ MRI, optical fluorescence imaging, and photoacoustic imaging [29-32]. PCCAs normally consist of droplets of liquid PFCs with boiling points near body temperature, such as dodecafluoropentane (b.p. $29^{\circ} \mathrm{C}$ ) or perfluorohexane (b.p. $60^{\circ} \mathrm{C}$ ), encapsulated in a lipid or polymer shell to optimize emulsion stability. The Clausius-Clapeyron relation dictates that the phase-transition temperature of a compound is inversely proportional to the pressure exerted on it. As droplet size diminishes, the Laplace pressure exerted on the core due to radial curvature increases. Therefore, PCCA droplets with micro- and nanoscale diameters may experience several atmospheres of pressure in addition to ambient pressure on the PFC core and the 'effective' boiling point is increased significantly. Using the Clausius-Clapeyron-derived Antoine vapor pressure equation, Sheeran et al. recently proposed that the substantial increase in Laplace pressure may allow for the generation of nanodroplets from decafluorobutane (DFB, b.p. $=-1.7^{\circ} \mathrm{C}$ ) [33]. The resulting droplets would, in theory, require less ultrasound energy to trigger vaporization than droplets composed of the most commonly-used PFCs - reducing the chance of unwanted ultrasound-induced bioeffects. Proof-of-concept was demonstrated with 
phospholipid-stabilized microscale DFB droplets, which showed significantly reduced vaporization thresholds compared to dodecafluoropentane and perfluorohexane.

Our group has recently demonstrated a unique technique of 'microbubble condensation' that allows for the ability to generate nanoscale decafluorobutane PCCAs in a simple manner [34]. By exposing pre-formed DFB UCAs to decreased ambient temperature and increased ambient pressure, a condensation of the perfluorocarbon core occurs (Figure 1). As the DFB core condenses, the increased Laplace pressure allows the droplets to remain in the liquid state when exposed to the original ambient temperature and pressure until vaporization is induced by thermal or acoustic energy.

Although it has been relatively unexplored in the literature, there is expected to be a tradeoff between the stability of the resulting emulsions and the acoustic pressure required to induce vaporization. Therefore, design of PCCAs from low boiling point perfluorocarbons for biomedical applications requires the ability to balance the overall stability through manipulation of factors such as 'effective' PFC boiling point and droplet size. In this study, we explore the ability of the microbubble condensation technique to generate PCCAs from perfluorocarbons with even lower boiling points than DFB, such as octafluoropropane (OFP, b.p. $-36.7^{\circ} \mathrm{C}$ ), and the tradeoff between relative in vitro stability and vaporization thresholds at both room and body temperature that results. Furthermore, we examine whether the balance between thermal stability and sensitivity to ultrasound-induced activation can be further manipulated by mixing different species of gaseous PFCs to a desired ratio prior to condensation. This concept is similar to one proposed by Kawabata and colleagues for liquid perfluorocarbons [35], however, it has not been previously shown to be possible with low boiling point, gaseous PFCs. Finally, we investigate whether the encapsulating lipid shell is preserved during the condensation process. Many groups have explored the ability to modify the shell of gas-core UCAs to provide a means of tissue-specific targeting or drug/gene delivery $[18,20,36]$. If the shell of condensed droplets remains after condensation, there is promise for extending nanodroplets composed of low boiling point PFCs to new diagnostic/ therapeutic functionalities simply by preparing the originating microbubbles with welldescribed methods.

\section{Methods}

\subsection{Microbubble Preparation}

Polydisperse decafluorobutane and octafluoropropane microbubbles were formulated by dissolution of 1,2-distearoyl-sn-glycero-3-phosphocholine (DSPC) and 1,2-distearoyl-snglycero-3-phosphoethanolamine-N-methoxy(polyethylene-glycol)-2000 (DSPE-PEG2000) in a 9:1 molar ratio and a total lipid concentration of $1.0 \mathrm{mg} / \mathrm{mL}$. All lipids were purchased from Avanti Polar Lipids (Alabaster, AL) and perfluorocarbons purchased from Fluoromed (Round Rock, TX). The excipient solution was comprised of phosphate-buffered saline (PBS), propylene glycol, and glycerol (16:3:1). After adding $1.5 \mathrm{~mL}$ of the resulting solution to a $3 \mathrm{~mL}$ glass vial, the headspace of the vial was gas-exchanged with each perfluorocarbon. Samples containing a mixture of perfluorocarbons were prepared by first filling a $30 \mathrm{~mL}$ syringe with DFB and OFP in a 1:1 ratio and then conducting a gas-exhange of the headspace of a lipid-containing $3 \mathrm{~mL}$ glass vial with the perfluorocarbon mixture. Microbubbles were formed via standard agitation techniques using a Vialmix shaker (Bristol-Myers-Squibb, New York, NY).

\subsection{Microbubble Condensation}

2.2.1. Condensation Procedure-The $3 \mathrm{~mL}$ vials containing PFC microbubble samples were immersed in a $\mathrm{CO}_{2}$ /isopropanol bath controlled to a temperature between $-5^{\circ} \mathrm{C}$ and 
$-10^{\circ} \mathrm{C}$ and swirled gently for approximately 1 minute. The vials were connected to an adjustable high-pressure air source and headspace pressure in the vial was increased until a change in consistency was noted in the microbubble sample - indicating the onset of condensation. The combination of propylene glycol, glycerol, and PBS prevented sample freezing during the short exposure to reduced temperatures (a total of approximately 2 minutes). After condensation, the pressure source was removed from the vial, leaving a pressure head on the solution until further use.

\subsection{Sample Sizing}

Microbubble sample size distributions were measured using an Accusizer 780 (Particle Sizing Systems, Santa Barbara, CA), which was capable of measuring particles as small as $0.5 \mu \mathrm{m}$ in diameter. Sample statistics were measured and averaged for 3 samples of each polydisperse group (DFB only, OFP only, 1:1 DFB + OFP). Accusizer sample volumes were typically on the order of $3 \mu \mathrm{L}$ for bubble samples. Dynamic light scattering (Malvern Nano ZS, Malvern Instruments Ltd., Malvern, Worcestershire, U.K.) was used to characterize content at the nanoscale for droplet samples. Approximately $1.5 \mathrm{~mL}$ of each condensed droplet sample was transferred to a cuvette by micropipette and the undiluted contents sized for each. The Malvern Nano ZS was capable of measuring particles up to $6 \mu \mathrm{m}$ in diameter.

\subsection{Sample Stability}

To determine the relative stability of PFC droplet emulsions, samples were transferred by micropipette to a $3 \mathrm{~mL}$ syringe and capped with no gas headspace. The syringes were exposed to either room temperature $\left(22^{\circ} \mathrm{C}\right)$ or body temperature $\left(37^{\circ} \mathrm{C}\right)$ by submerging the syringe in a heated water bath. Instability in the sample due to ambient temperature should manifest as the spontaneous phase-transition of the largest droplets in the sample (the least thermally stable), resulting in bubbles at the microscale. To capture this instability, the samples were sized using the Accusizer 780A at 10-minute intervals over the course of an hour to determine changes in the size distribution as well as concentration. Three samples of each group (DFB only, OFP only, 1:1 DFB + OFP) were measured at each temperature and the resulting distributions averaged. To display the change in concentration concurrently, the distribution at each time point was normalized and weighted relative to the peak concentration over the entire test.

\subsection{Ultrasonic Vaporization Thresholds}

2.5.1. Experimental Apparatus-The acoustic setup used for these experiments was similar to that described in earlier studies [33]. Briefly, an acrylic-lined, continuously degassed water bath was mounted onto an inverted microscope (Olympus IX71, Center Valley, PA). Depending on the experiment, the water bath temperature was either maintained at $22^{\circ} \mathrm{C}$ or $37^{\circ} \mathrm{C}$. The $100 \times(\mathrm{NA}=1.0)$ water immersion objective was interfaced with a high-speed camera (FastCam SA1.1, Photron USA, Inc., San Diego, CA). Images and videos captured during the experiments were analyzed using ImageJ software $(\mathrm{NIH}$, Bethesda, MD). The optical resolution of the system limited measurement of particles to those greater than or equal to approximately $1 \mu \mathrm{m}$ in diameter to maintain reasonable accuracy. Solutions of droplets and bubbles were pumped through a nearly optically and acoustically transparent cellulose tube with a $200 \mu \mathrm{m}$ inner diameter (Spectrum Laboratories, Inc., Greensboro, NC) using a custom-built manual injector that allowed for precise spatial manipulation of the sample volume observed. The portion of the tube in focus was manipulated by a 3-axis micropositioner (MMO-203, Narishige Group, East Meadow, NY).

A spherically-focused transducer with a $7.5 \mathrm{MHz}$ center frequency and focal length of $\sim 5$ $\mathrm{cm}$ (V321, Panametrics, Inc., Waltham, MA) was used to transmit acoustic pulses into 
droplet samples. Signals consisting of a 2-cycle sinusoid of adjustable amplitude at $8 \mathrm{MHz}$ (total insonification time of $250 \mathrm{~ns}$ ) were provided using an arbitrary waveform generator (AFG 3101, Tektronix, Inc., Beaverton, OR) and amplified approximately $60 \mathrm{~dB}$ using an RF amplifier (A500, ENI, Rochester, NY) before being delivered to the transducer. For optical-acoustic alignment, the focus of the transducer was positioned to be confocal with the microscope objective focus by a calibrated needle hydrophone (HNA-0400, Onda Corp., Sunnyvale, CA). The range of transducer output pressures was calibrated at the focus to determine the approximate pressures experienced by droplets in the field of view.

2.5.2. Microscale Droplet Vaporization Threshold-Based on preliminary studies involving microbubble condensation [34], the majority of the droplets in each sample have diameters in the hundreds of nanometers. However, the presence of optically resolvable microscale outlier droplets enables simple measurement of individual droplet vaporization thresholds, which is convenient for comparison of the thresholds between different perfluorocarbons. Samples were first vented with a $20 \mathrm{G}$ needle and diluted in PBS until only a few droplets were visible on screen at any particular time. This was typically on the order of 10X dilution, but varied from sample to sample based on the number of outlier droplets present. The large outlier droplets, which rested near the bottom of the microcellulose tube, were maintained in focus using the position manipulator. Ultrasound pulses were delivered in pressure increments of approximately $55 \mathrm{kPa}$ (rarefactional) with approximately $1-2 \mathrm{~s}$ of rest time between each manual pulse trigger. The rarefactional pressure that induced phase-transition in the droplet was recorded for correlation with droplet diameter.

\subsection{Shell Preservation}

To create microbubbles incorporating fluorescent marker in the lipid shell, $2 \mu \mathrm{L}$ of Vybrant DiI cell-labeling solution (Invitrogen Molecular Probes, Eugene, OR) was added to the previously described lipid solution. The headspace of the vial was gas-exchanged with DFB and subsequently agitated to form microbubbles. Condensation of the microbubble sample was performed with the techniques described above. Brightfield and fluorescent digital micrographs were obtained before microbubble condensation using a 100X objective with a fluorescence microscope (BX51, Olympus, Center Valley, PA) and Metamorph Basic software (Molecular Devices, Sunnyvale, CA).

\section{Results}

\subsection{Microbubble and Droplet Size Distributions}

All microbubble samples showed similar distribution characteristics regardless of which perfluorocarbon or perfluorocarbon mixture was selected for the core (Table 1), although there was a significant increase in mean concentration for the 1:1 DFB + OFP microbubbles compared to DFB microbubbles ( $p=0.01$ by student's 2 -sided unpaired t-test), and a significant decrease in size for 1:1 DFB + OFP microbubbles compared to DFB and OFP microbubbles ( $p=0.01$ and $p=0.05$, respectively).

\subsection{Droplet Size Distribution}

Dynamic light scattering (DLS) showed that the decrease in the size of the particles after condensation resulted in droplet distributions primarily in the sub-micron range, which is consistent with preliminary studies [34]. Samples containing DFB appeared to condense with very little additional pressure (4-5 psi), while the 1:1 DFB + OFP mixture samples and pure OFP samples required substantially more (25-30 psi and 45-50 psi, respectively). Nanodroplets originating from DFB microbubble samples and 1:1 DFB + OFP mixture microbubble samples both condensed to form peaks at $295 \mathrm{~nm}$ (Figure 2). The DFB 
nanodroplets had slightly higher average diameters than the samples created from a 1:1 PFC mixture (363 $\pm 161 \mathrm{~nm}$ and $296 \pm 94 \mathrm{~nm}$, respectively). Pure OFP samples did not show sufficient stability to be sized by DLS, although the optical data and vaporization data (see below) confirms the condensation of OFP into both microscale and nanoscale droplets.

\subsection{Droplet Emulsion Stability}

The content of each sample at the beginning of the 60-minute test period showed that the mode particle size was near the lower sensitivity threshold of the Accusizer. This confirms that the actual size distribution of the sample is best represented by the sub-micron sizing (DLS). The Accusizer, however, allows for simple characterization of the change in distribution and concentration over time of the content greater than $500 \mathrm{~nm}$ in diameter. Because the largest content in the phase-change emulsions is the least stable, the greatest change in distribution and concentration over time should occur in the upper portion of the sample distribution.

DFB exhibited remarkable stability in vitro at both temperatures, with a preservation of distribution characteristics (Figure 3a,b) and no significant change in mean concentration (Figure 4a,b) over the period. The PFC mixture samples showed a general preservation of distribution characteristics (Figure 3c), but a 17\% drop in mean concentration over the time period at $22^{\circ} \mathrm{C}(p=0.05$, Figure $4 a)$. At $37^{\circ} \mathrm{C}$, the PFC mixture samples showed a $45 \%$ decrease in mean concentration over the test period $(p=0.03$, Figure $4 b)$, and an increase in the content between 3-10 $\mu \mathrm{m}$ was observed in the distribution profile (Figure $3 \mathrm{~d}$ ). OFP samples showed significant instability at both temperatures. At both $22^{\circ} \mathrm{C}$ and $37^{\circ} \mathrm{C}$, an $82 \%$ decrease in mean concentration was observed over the time period $(p=0.04$ and $p=0.01$, respectively, Figure $4 a, b)$, although the decrease in concentration was more gradual over time in the $22^{\circ} \mathrm{C}$ case. At $37^{\circ} \mathrm{C}$, OFP samples decrease to their final concentration with the first 10-20 minutes, and a corresponding increase in the content between 3 and $10 \mu \mathrm{m}$ was observed in the distribution profile (Figure 3f).

\section{Droplet Vaporization Thresholds}

3.3.1. Microscale Droplet Thresholds: Diluting droplet samples in PBS allowed microscale outlier droplets to be isolated and vaporized individually (Figure 5). By exposing 20-30 droplets of each PFC to acoustic pressure, the peak rarefactional pressure that initiated droplet vaporization could be mapped against droplet diameter to observe general trends in vaporization thresholds (Figures 6 and 7). Results show that both perfluorocarbon boiling point and ambient temperature greatly influenced the rarefactional pressure needed to vaporize droplets, as expected, and that the vaporization pressure generally increased with decreasing diameter in the small range tested. Additionally, a droplet core composed of a mixture of perfluorocarbons resulted in droplets with vaporization thresholds between each of the 'pure' perfluorocarbons (Figure 7).

3.3.2. Shell Preservation: Brightfield and fluorescence microscopy results showed that the DiI-labeled encapsulating lipid shell was present after the condensation technique (Figure 8). Although this can be expected, it has not been previously illustrated, and is important for future incorporation of therapeutic and targeting agents.

\section{Discussion}

This report demonstrates the extent to which a new class of low boiling point perfluorocarbons can be manipulated with regard to stability and vaporization thresholds in order to design phase-change ultrasound contrast agents with desirable properties. Perhaps the most surprising result of these studies was that OFP, which comprises the PFC gas-core 
of the commercial UCA Definity ${ }^{\mathrm{TM}}$ (Lantheus Medical Imaging, Inc., USA) [11], can be condensed to form droplets that exhibit some stability at $22{ }^{\circ} \mathrm{C}$ - nearly $60{ }^{\circ} \mathrm{C}$ above its normal boiling point of $-36.7^{\circ} \mathrm{C}$. Furthermore, OFP droplets as large as $5-8 \mu \mathrm{m}$ were able to be optically resolved and vaporized (Figure 7). Once exposed to physiological temperatures, however, the upper distribution of OFP emulsions represented by the Accusizer show rapid spontaneous vaporization. While this may be unfavorable for most PCCA applications where only ultrasonic activation is desired, it may be useful for creating ultrasound contrast agents that vaporize immediately upon injection.

Both the sizing and concentration data over the course of the 1-hour test period revealed the remarkable stability of DFB droplets when exposed to both room and body temperature in vitro. In contrast, the portion of the OFP samples registered by the Accusizer were greatly sensitive to both room and body temperature - showing rapid changes in the size distributions and in concentration. Samples composed of 1:1 mixtures of OFP and DFB showed generally favorable stability at room temperature with regard to both distribution and concentration. Once exposed to body temperature, the samples retained the distribution profiles, although the concentration dropped at a much faster rate than at room temperature. These results highlight the expected relationship between general thermal stability and PFC boiling point, including the effect of modulating the boiling point by mixing perfluorocarbons. The thermal stability of droplets with diameters smaller than $500 \mathrm{~nm}$ (not registered by the Accusizer) for all PFC compositions requires further investigation. For example - while the portion of OFP samples greater than $500 \mathrm{~nm}$ appeared generally unstable, it is possible that droplets in the $100-300 \mathrm{~nm}$ range may be sufficiently stable for use at body temperature as a result of the increased Laplace overpressure. If so, then future refinement of the size distribution to isolate this small content along with optimization of the shell composition may sufficiently stabilize OFP samples to remain in the liquid state upon injection. Additionally, the droplet samples in the present study were measured under atmospheric pressure, and so the higher in vivo pressures may further stabilize circulating and extravasated droplets.

A common convention in measuring acoustic output is to normalize the peak rarefactional pressure (in MPa) by the square root of the US frequency (in $\mathrm{MHz}$ ) - termed the 'mechanical index' (MI). Typical clinical diagnostic ultrasound machines are designed with a maximum MI of 1.9 or less to reduce the likelihood of inertial cavitation and unwanted bioeffects, although it is suggested that contrast-enhanced diagnostic imaging be performed at much lower MIs. In this study, all microscale droplets were vaporizable at both room temperature and body temperature using very short pulses ( 2 cycles) and with mechanical indices less than 1.9 (approximately equivalent to $5.4 \mathrm{MPa}$ at $8 \mathrm{MHz}$ ). The trends confirm the inverse relationship between PFC boiling point and droplet vaporization threshold as well as the expected inverse relationship between ambient temperature and droplet vaporization threshold. Microscale DFB droplets were vaporized at pressures near $2 \mathrm{MPa}$ (MI of approximately 0.71 ) at body temperature (Figure 6), which is nearly 30\% lower than the pressures needed to vaporize at $5 \mathrm{MHz}$ obtained from a previous study [33]. Although the acoustic pulse lengths were slightly different in this study than the previous, decreasing vaporization pressure as a function of increasing US frequency has been reported previously in the literature and is confirmed here [3,37]. Microscale OFP droplets could be vaporized with pressures on the order of $0.5 \mathrm{MPa}$ (MI of approximately 0.18 ) at body temperature. In many applications, it may be preferable to perform investigatory imaging at low pressures and vaporize injected PCCAs at a desired point using an 'activation pulse' of higher pressure. For these purposes, the low vaporization threshold of microscale OFP droplets may be unsuitable, and droplets composed of a mixture of PFCs or pure DFB may be more appropriate. 
The concept of mixing liquid perfluorocarbons to modulate emulsion properties was initially proposed by Kawabata and colleagues [35]. This study is the first to show that stability and activation energy of emulsions can be modified by applying a similar concept to gaseous perfluorocarbons prior to microbubble condensation. As shown in Figure 7, droplets composed of a mixture of PFCs were vaporized at pressures between those required to vaporize 'pure' droplets of either OFP or DFB, similar to the stability characteristics observed (Figures 3 and 4). Although a significant amount of variation is present in the thresholds, no overlapping points occurred between the perfluorocarbon groups, which suggests that the PFCs were miscible and could be condensed to form a mixed droplet.

It is expected that the acoustic field near the bottom of the microcellulose tube is complex due to interaction with the tube itself [38]. This may explain some of the variability present in the droplet vaporization thresholds, as the microscale droplets rested on the tube prior to vaporization. It may also be possible that the tube damped the pressure experienced by the droplets, and that the actual pressures used to vaporize were lower than those reported here. Additionally, it was noted during testing that very slight changes in transducer position could yield significant changes in the reported vaporization threshold for microscale droplets. This variation in transducer alignment can be seen in the values reported for both DFB and OFP droplets at room temperature in Figures 6 and 7, where the trends appear to be shifted upward to some degree in the latter image because they were collected under a slightly different alignment. To ensure this source of variation did not influence conclusions drawn regarding the efficacy of PFC mixing or relative vaporization energy, each figure represents data collected on the same day under the same alignment conditions.

Measuring droplet vaporization thresholds for microscale droplets is convenient to compare the general influence of factors such as PFC choice and temperature. Evaluating appropriate vaporization pressures for nanoscale droplets, on the other hand, is confounded by their inability to be individually resolved optically. In this study, the presence of microscale OFP droplets implies the presence of nanoscale OFP droplets, although the samples were too unstable to prove this by DLS. To verify the expected presence of vaporizable nanoscale OFP droplets, a sample of OFP droplets was diluted 50\% in PBS and exposed to an acoustic rarefactional pressure of $1.1 \mathrm{MPa}$ (MI of approximately 0.4 ) at $37^{\circ} \mathrm{C}$ (Figure 9). This pressure was selected based on being approximately twice the pressure required to vaporize microscale droplets. The resulting bubbles, which were between $1-5 \mu \mathrm{m}$, confirm the presence of vaporizable sub-micron OFP droplets as small as $200-300 \mathrm{~nm}$ by ideal gas law estimations [33, 39]. Future studies will be needed to more precisely define the appropriate pressure levels needed to activate sub-micron content present in the samples both in vitro and in vivo.

Results showing preservation of the lipid shell demonstrate the possibility to simply incorporate elements such as tissue-specific targeting ligands, genes, or drugs by including them at the microbubble state and then condensing to form the modified droplets. Few studies to date have explored PCCAs capable of gene delivery or active tissue targeting prior to vaporization. In our initial studies of DFB nanodroplets, a cationic lipid shell was used that could be made to carry gene vectors [34]. Incorporating ligands that target cellular markers of angiogenesis could result in ultrasonically-activatable nanodroplets capable of molecular imaging of the extravascular space.

The size of a PCCA also plays a key role in the relationship between thermal stability and sensitivity to ultrasound. Previously described methods of controlling or altering size distributions of PFC droplets have relied on microfluidic sorting, microfluidic droplet generation, or filtering [3, 30, 40-44]. Because the size of a PFC droplet produced by microbubble condensation is largely dependent on the initial microbubble size, the size 
distribution of PFC droplets could be tailored as desired by simply controlling the size distribution of the initial microbubble samples through well-described techniques such as differential centrifugation or microfluidics $[45,46]$. Combining precursor microbubble sizeselection with PFC mixing may provide a means of highly-tuning droplet sample characteristics to specific applications.

\section{Conclusion}

In this study, we have demonstrated proof-of-principle that it is possible to design PCCAs from a variety of low boiling point PFCs that have not been previously considered. The results show that a given droplet's balance between stability and 'vaporizability' is a tradeoff of several factors such as PFC composition and ambient temperature. Through the methods presented and discussed here, it is conceivable that one could customize the droplet characteristics (size, thermal stability, vaporization threshold) with a particular application in mind. These results demonstrate the promise for low boiling point PFCs as a useful new class of compounds for activatable contrast agents.

\section{Acknowledgments}

This work was supported by NIH grant no. EB-011704. The authors acknowledge Professor Mark Borden at the University of Colorado for insightful discussions regarding microbubble condensation, Steven Feingold for assistance with calibrations, and Drs. Russell Mumper and Michael Jay in the UNC Eshelman School of Pharmacy for assistance with DLS sizing. P.S. appreciates the generous support of the National Science Foundation as the recipient of a graduate fellowship.

\section{References}

1. Kim T, Huh YM, Haam S, Lee K. Activatable nanomaterials at the forefront of biomedical sciences. J Mater Chem. 2010; 20(38):8194-8206.

2. Kripfgans OD, Fowlkes JB, Miller DL, Eldevik OP, Carson PL. Acoustic droplet vaporization for therapeutic and diagnostic applications. Ultrasound Med Biol. 2000; 26(7):1177-1189. [PubMed: 11053753]

3. Kripfgans OD, Fowlkes JB, Woydt M, Eldevik OP, Carson PL. In vivo droplet vaporization for occlusion therapy and phase aberration correction. IEEE Trans Ultrason Ferroelectr Freq Control. 2002; 49(6):726-738. [PubMed: 12075966]

4. Fabiilli ML, Lee JA, Kripfgans OD, Carson PL, Fowlkes JB. Delivery of water-soluble drugs using acoustically triggered perfluorocarbon double emulsions. Pharm Res. 2010; 27(12):2753-2765. [PubMed: 20872050]

5. Miller DL, Kripfgans OD, Fowlkes JB, Carson PL. Cavitation nucleation agents for nonthermal ultrasound therapy. J Acoust Soc Am. 2000; 107(6):3480-3486. [PubMed: 10875392]

6. Zhang M, Fabiilli ML, Haworth KJ, Padilla F, Swanson SD, Kripfgans OD, et al. Acoustic droplet vaporization for enhancement of thermal ablation by high intensity focused ultrasound. Acad Radiol. 2011; 18(9):1123-1132. [PubMed: 21703883]

7. Campbell RB. Tumor physiology and delivery of nanopharmaceuticals. Anticancer Agents Med Chem. 2006; 6(6):503-512. [PubMed: 17100555]

8. Rapoport NY, Kennedy AM, Shea JE, Scaife CL, Nam KH. Controlled and targeted tumor chemotherapy by ultrasound-activated nanoemulsions/microbubbles. J Control Release. 2009; 138(3):268-276. [PubMed: 19477208]

9. Zhang P, Porter T. An in vitro study of a phase-shift nanoemulsion: a potential nucleation agent for bubble-enhanced HIFU tumor ablation. Ultrasound Med Biol. 2010; 36(11):1856-1866. [PubMed: 20888685]

10. Wang CH, Kang ST, Lee YH, Luo YL, Huang YF, Yeh CK. Aptamer-conjugated and drug-loaded acoustic droplets for ultrasound theranosis. Biomaterials. 2012; 33(6):1939-1947. [PubMed: 22142768] 
11. Lantheus Medical Imaging. Definity ${ }^{\circledR}$ (perflutren lipid microsphere) injectable suspension. N Billerica, MA: Lantheus Medical Imaging; 2011. Online, Available from URL: http://www.definityimaging.com/main.html

12. Porter, TR. Ultrasound contrast agents and methods for their manufacture and use. US Patent. No 5567415. 1996.

13. Mullin L, Gessner R, Kwan J, Kaya M, Borden MA, Dayton PA. Effect of anesthesia carrier gas on in vivo circulation times of ultrasound microbubble contrast agents in rats. Contrast Media Mol Imaging. 2011; 6(3):126-131. [PubMed: 21246710]

14. Wei K, Mulvagh SL, Carson L, Davidoff R, Gabriel R, Grimm RA, et al. The safety of definity and optison for ultrasound image enhancement: a retrospective analysis of 78,383 administered contrast doses. J Am Soc Echocardiogr. 2008; 21(11):1202-1206. [PubMed: 18848430]

15. Porter TR, Xie F. Transient myocardial contrast after initial exposure to diagnostic ultrasound pressures with minute doses of intravenously injected microbubbles: demonstration and potential mechanisms. Circulation. 1995; 92(9):2391-2395. [PubMed: 7586336]

16. Wei K, Jayaweera AR, Firoozan S, Linka A, Skyba DM, Kaul S. Quantification of myocardial blood flow with ultrasound-induced destruction of microbubbles administered as a constant venous infusion. Circulation. 1998; 97(5):473-483. [PubMed: 9490243]

17. Klibanov AL. Microbubble contrast agents: targeted ultrasound imaging and ultrasound-assisted drug-delivery applications. Invest Radiol. 2006; 41(3):354-362. [PubMed: 16481920]

18. Gessner R, Dayton PA. Advances in molecular imaging with ultrasound. Mol Imaging. 2010; 9(3): 117-127. [PubMed: 20487678]

19. Chomas JE, Pollard RE, Sadlowski AR, Griffey SM, Wisner ER, Ferrara KW. Contrast-enhanced US of microcirculation of superficially implanted tumors in rats. Radiology. 2003; 229(2):439446. [PubMed: 14526091]

20. Ferrara K, Pollard R, Borden M. Ultrasound microbubble contrast agents: fundamentals and application to gene and drug delivery. Annu Rev Biomed Eng. 2007; 9:415-447. [PubMed: 17651012]

21. Tachibana K, Tachibana S. Albumin microbubble echo-contrast material as an enhancer for ultrasound accelerated thrombolysis. Circulation. 1995; 92(5):1148-1150. [PubMed: 7648659]

22. Tran BC, Seo J, Hall TL, Fowlkes JB, Cain CA. Microbubble-enhanced cavitation for noninvasive ultrasound surgery. IEEE Trans Ultrason Ferroelectr Freq Control. 2003; 50(10):1296-1304. [PubMed: 14609069]

23. Stride EP, Coussios CC. Cavitation and contrast: the use of bubbles in ultrasound imaging and therapy. Proc Inst Mech Eng H. 2010; 224(H2):171-191. [PubMed: 20349814]

24. Hall CS, Lanza GM, Rose JH, Kaufmann RJ, Fuhrhop RW, Handley SH, et al. Experimental determination of phase velocity of perfluorocarbons: applications to targeted contrast agents. IEEE Trans Ultrason Ferroelectr Freq Control. 2000; 47(1):75-84. [PubMed: 18238519]

25. Pisani E, Tsapis N, Paris J, Nicolas V, Cattel L, Fattal E. Polymeric nano/microcapsules of liquid perfluorocarbons for ultrasonic imaging: physical characterization. Langmuir. 2006; 22(9):43974402. [PubMed: 16618193]

26. Mattrey RF. Perfluorooctylbromide: a new contrast agent for CT, sonography, and MR imaging. AJR Am J Roentgenol. 1989; 152(2):247-252. [PubMed: 2643258]

27. Quay, SC. Phase shift colloids as ultrasound contrast agents. US Patent. No 5558853. 1996.

28. Apfel, RE. Activatable infusable dispersions containing drops of a superheated liquid for methods of therapy and diagnosis. US Patent. No 5840276. 1998.

29. Rapoport N, Nam KH, Gupta R, Gao Z, Mohan P, Payne A, et al. Ultrasound-mediated tumor imaging and nanotherapy using drug loaded, block copolymer stabilized perfluorocarbon nanoemulsions. J Control Release. 2011; 153(1):4-15. [PubMed: 21277919]

30. Couture O, Faivre M, Pannacci N, Babataheri A, Servois V, Tabeling P, et al. Ultrasound internal tattooing. Med Phys. 2011; 38(2):1116-1123. [PubMed: 21452748]

31. Strohm E, Rui M, Gorelikov I, Matsuura N, Kolios M. Vaporization of perfluorocarbon droplets using optical irradiation. Biomed Opt Express. 2011; 2:1432-1442. [PubMed: 21698007] 
32. Rajian JR, Fabiilli ML, Fowlkes JB, Carson PL, Wang X. Drug delivery monitoring by photoacoustic tomography with an ICG encapsulated double emulsion. Opt Express. 2011; 19(15): 14335-14347. [PubMed: 21934797]

33. Sheeran PS, Wong VP, Luois S, McFarland RJ, Ross WD, Feingold S, et al. Decafluorobutane as a phase-change contrast agent for low-energy extravascular ultrasonic imaging. Ultrasound Med Biol. 2011; 37(9):1518-1530. [PubMed: 21775049]

34. Sheeran PS, Luois S, Dayton PA, Matsunaga TO. Formulation and acoustic studies of a new phase-shift agent for diagnostic and therapeutic ultrasound. Langmuir. 2011; 27(17):10412-10420. [PubMed: 21744860]

35. Kawabata KI, Sugita N, Yoshikawa H, Azuma T, Umemura SI. Nanoparticles with multiple perfluorocarbons for controllable ultrasonically induced phase shifting. Jpn J Appl Phys. 2005; 44(6B):4548-4552.

36. Hernot S, Klibanov AL. Microbubbles in ultrasound-triggered drug and gene delivery. Adv Drug Deliv Rev. 2008; 60(10):1153-1166. [PubMed: 18486268]

37. Schad KC, Hynynen K. In vitro characterization of perfluorocarbon droplets for focused ultrasound therapy. Phys Med Biol. 2010; 55(17):4933-4947. [PubMed: 20693614]

38. Qin S, Kruse DE, Ferrara KW. Transmitted ultrasound pressure variation in micro blood vessel phantoms. Ultrasound Med Biol. 2008; 34(6):1014-1020. [PubMed: 18395962]

39. Evans DR, Parsons DF, Craig VSJ. Physical properties of phase-change emulsions. Langmuir. 2006; 22(23):9538-9545. [PubMed: 17073477]

40. Giesecke T, Hynynen K. Ultrasound-mediated cavitation thresholds of liquid perfluorocarbon droplets in vitro. Ultrasound Med Biol. 2003; 29(9):1359-1365. [PubMed: 14553814]

41. Huh D, Bahng JH, Ling Y, Wei HH, Kripfgans OD, Fowlkes JB, et al. Gravity-driven microfluidic particle sorting device with hydrodynamic separation amplification. Anal Chem. 2007; 79(4): 1369-1376. [PubMed: 17297936]

42. Nieuwstadt HA, Seda R, Li DS, Fowlkes JB, Bull JL. Microfluidic particle sorting utilizing inertial lift force. Biomed Microdevices. 2010; 13(1):97-105. [PubMed: 20865451]

43. Bardin D, Martz TD, Sheeran PS, Shih R, Dayton PA, Lee AP. High-speed, clinical-scale microfluidic generation of stable phase-change droplets for gas embolotherapy. Lab Chip. 2011; 11:3990-3998. [PubMed: 22011845]

44. Martz TD, Sheeran PS, Bardin D, Lee AP, Dayton PA. Precision manufacture of phase-change perfluorocarbon droplets using microfluidics. Ultrasound Med Biol. 2011; 37(11):1952-1957. [PubMed: 21963036]

45. Feshitan JA, Chen CC, Kwan JJ, Borden MA. Microbubble size isolation by differential centrifugation. J Colloid Interface Sci. 2009; 329(2):316-324. [PubMed: 18950786]

46. Talu E, Hettiarachchi K, Zhao S, Powell RL, Lee AP, Longo ML, et al. Tailoring the size distribution of ultrasound contrast agents: possible method for improving sensitivity in molecular imaging. Mol Imaging. 2007; 6(6):384-392. [PubMed: 18053409] 


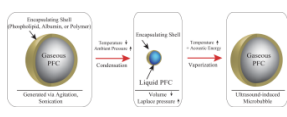

FIGURE 1.

Exposing pre-formed PFC microbubbles to decreased ambient temperature and increased ambient pressure results in condensation of the gaseous core. The decreased size results in an increased Laplace pressure, which serves to preserve the particle in the liquid state. Once exposed to increased temperature and energy delivered via ultrasound, vaporization of the droplet core results in a larger, highly echogenic gas microbubble. 


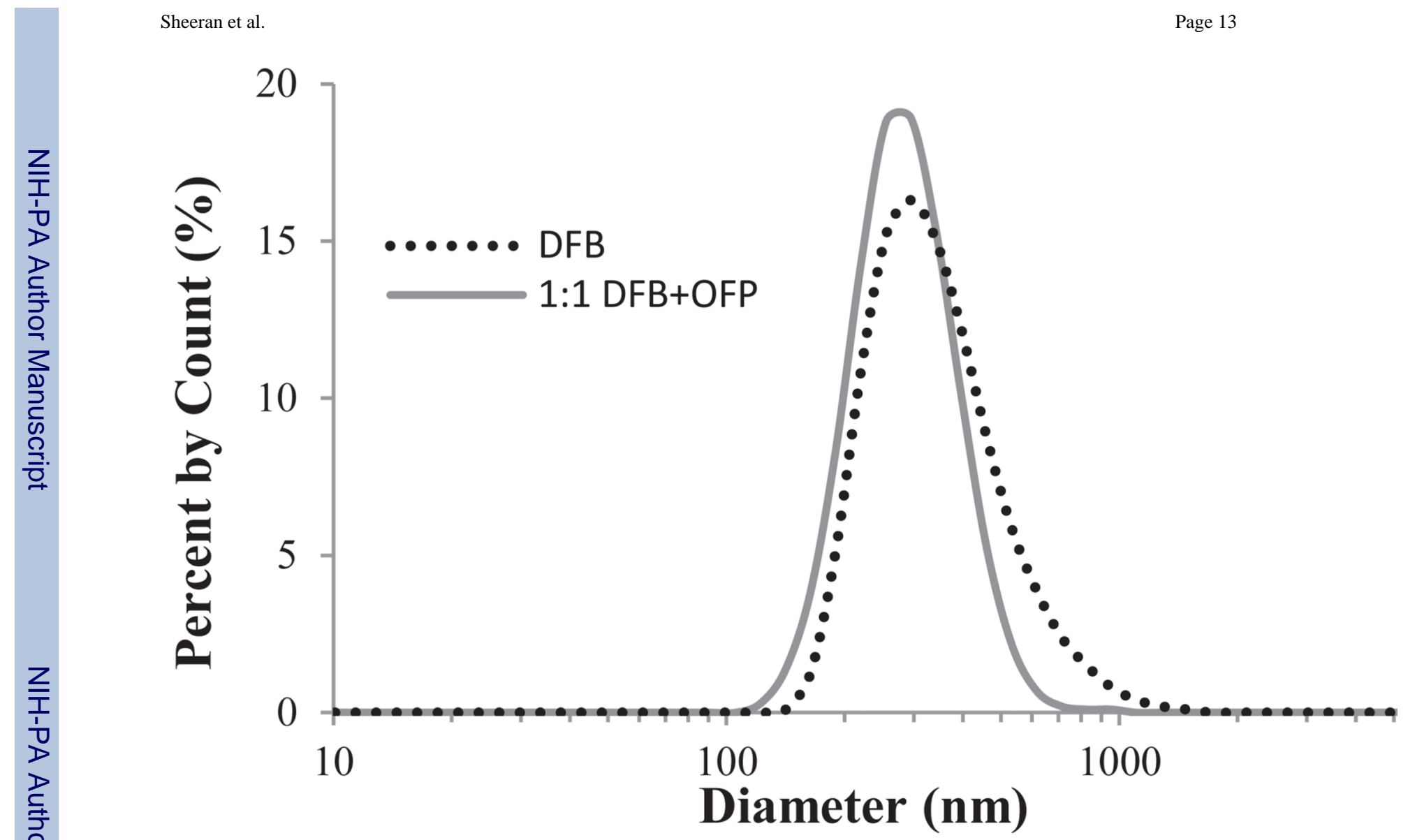

FIGURE 2.

Dynamic light scattering results for various nanodroplet formulations ( $\mathrm{N}=3$ for each group). Both DFB and 1:1 DFB + OFP droplets resulting from polydisperse bubbles showed peaks at $295 \mathrm{~nm}$ in diameter. 


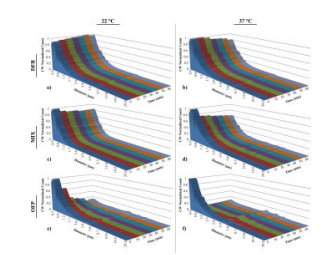

FIGURE 3.

Perfluorocarbon droplet distributions in vitro over a 1-hour period: Pure decafluorobutane at a) $22^{\circ} \mathrm{C}$ and b) $37^{\circ} \mathrm{C}$; DFB + OFP mixture at c) $22^{\circ} \mathrm{C}$ and d) $37^{\circ} \mathrm{C}$; and pure octafluoropropane at e) $22^{\circ} \mathrm{C}$ and d) $37^{\circ} \mathrm{C}$. The distribution at each timepoint was scaled to the relative mean concentration (concentration-weighted) to simultaneously reflect changes in concentration over the time period. 


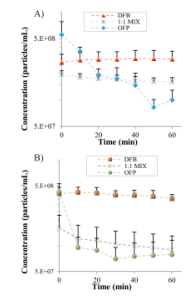

FIGURE 4.

Change in concentration over time for droplet samples of each perfluorocarbon at a) $22^{\circ} \mathrm{C}$ and b) $37^{\circ} \mathrm{C}$. 


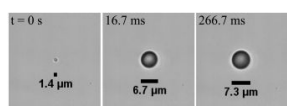

FIGURE 5.

A microscale droplet of OFP exposed to a $0.25 \mu$ s pulse at approximately $0.55 \mathrm{MPa}$ vaporizes to form a gas bubble approximately 5 -fold larger 


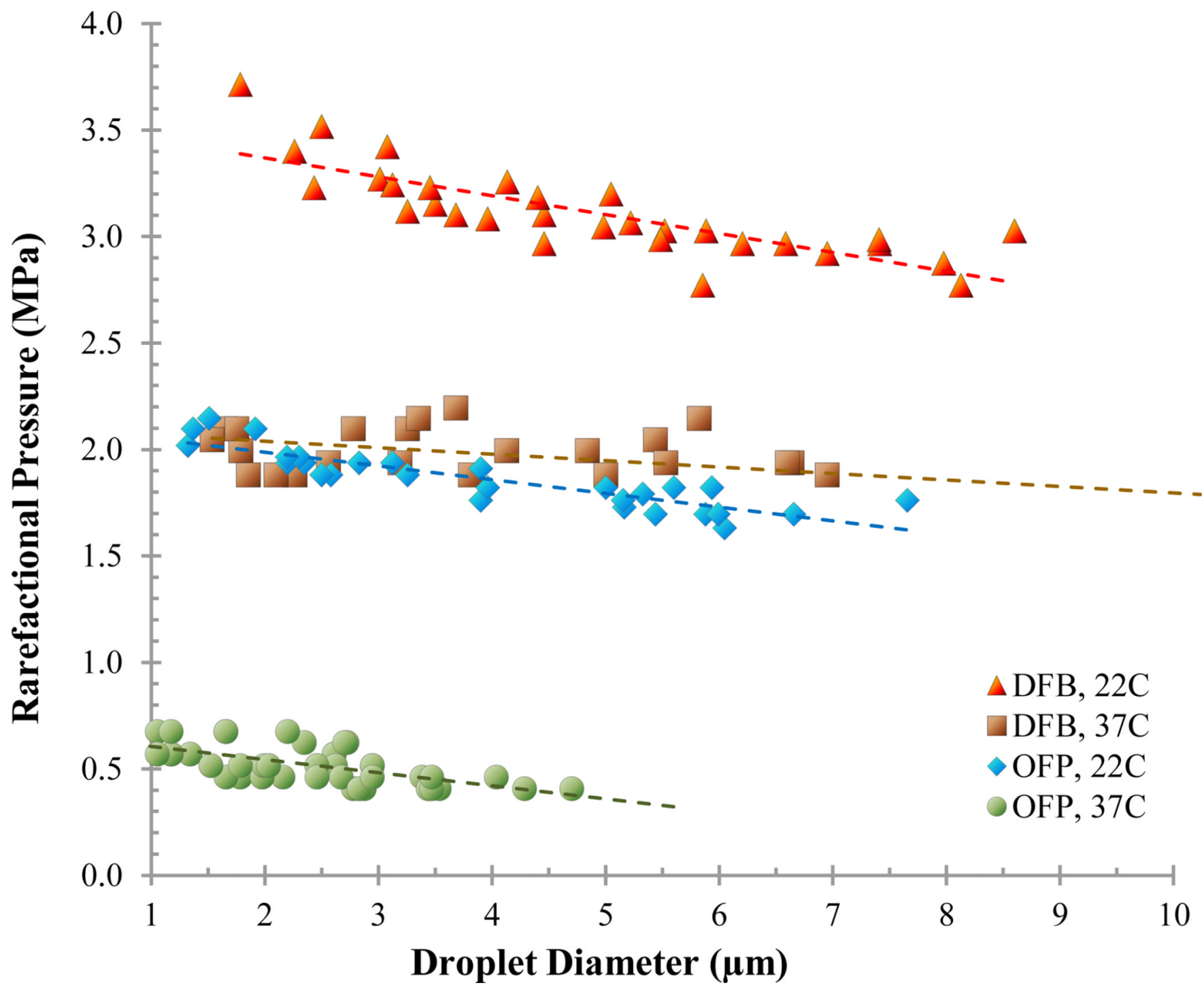

FIGURE 6.

Vaporization pressure for microscale DFB and OFP droplets at $22^{\circ} \mathrm{C}$ and $37^{\circ} \mathrm{C}$. The vaporization threshold increased with increasing boiling point and decreasing diameter for the small range observed. 


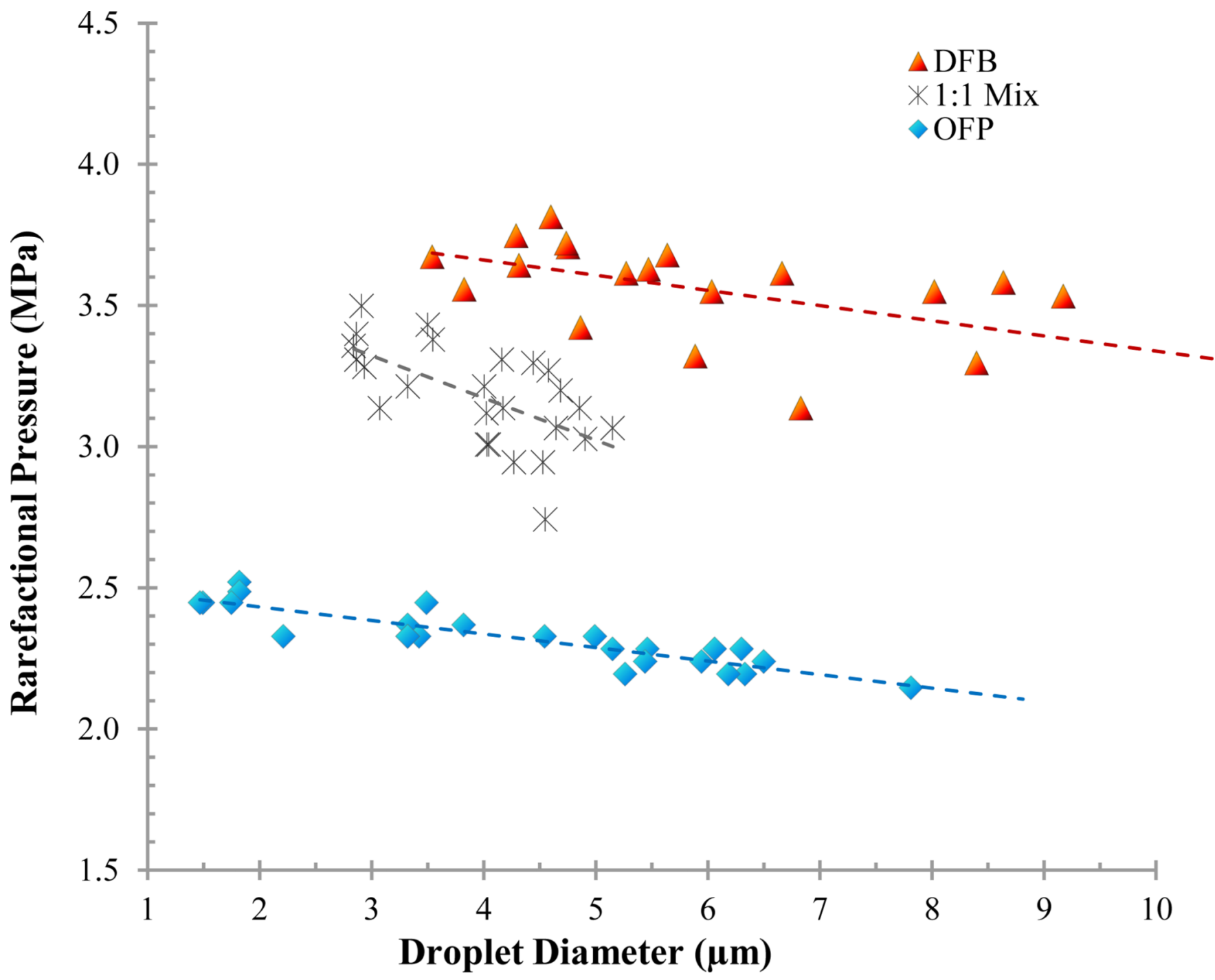

FIGURE 7.

Droplets composed of a mixture of PFCs vaporized at $22{ }^{\circ} \mathrm{C}$ with rarefactional pressures between each of the composing PFCs - indicating condensation of the mixed gases resulted in a miscible dual-PFC core. By adjusting the ratio of PFCs, the vaporization threshold may be further 'tuned'. 

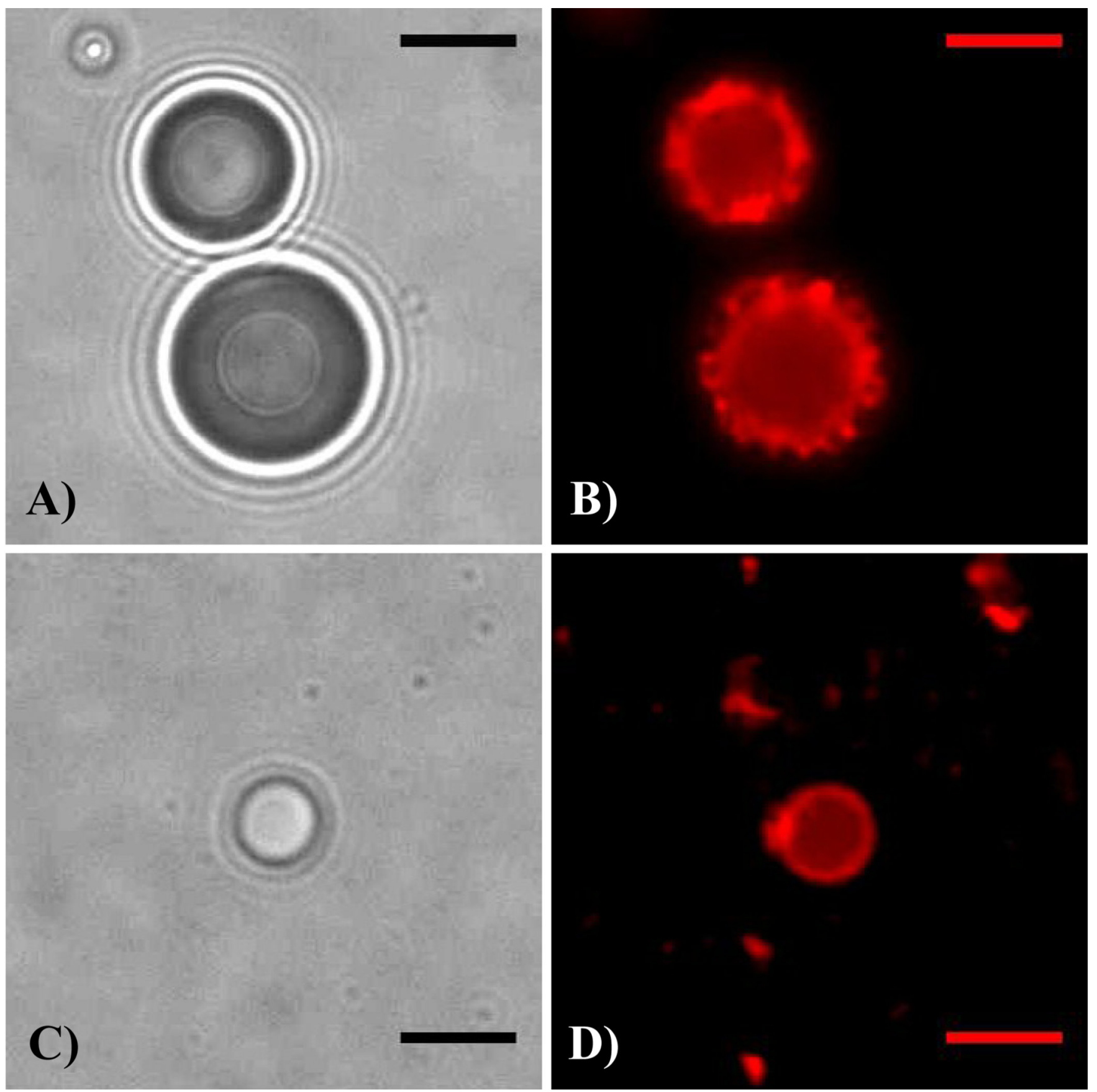

FIGURE 8.

Brightfield (a, c) and fluorescence (b, d) microscopy illustrating that the shell is preserved when DiI-labeled DFB microbubbles ( $a, b)$ are condensed to the liquid state (c, d), the shell is preserved through the change in volume. (note that the droplet in c-d did not result directly from condensation of the precursor bubbles in $\mathrm{a}-\mathrm{b}$ ). Scale bar is 5 micrometers. 


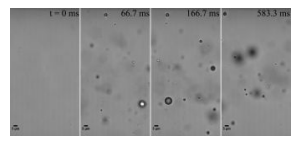

Figure 9.

OFP droplet sample exposed to a $0.250 \mu$ s pulses pulse at $1.1 \mathrm{MPa}$ initiated at $\mathrm{t}=0$ forms bubbles in the $1-5 \mu \mathrm{m}$ range, confirming the presence of viable nanoscale OFP droplets. 


\section{Table 1}

Microbubble Distribution Statistics

\begin{tabular}{lcc}
\hline Sample & $\begin{array}{c}\text { Mean Diam. } \pm \\
\text { S.D. }(\boldsymbol{\mu m})\end{array}$ & $\begin{array}{c}\text { Concentration } \\
(\text { particles/mL) }\end{array}$ \\
\hline DFB Only & $1.12 \pm 0.83$ & $8.93 \times 10^{9} \pm 2.28 \times 10^{9}$ \\
OFP Only & $1.10 \pm 0.81$ & $1.278 \times 10^{10} \pm 2.32 \times 10^{9}$ \\
DFB + OFP & $1.02 \pm 0.64$ & $1.582 \times 10^{10} \pm 2.73 \times 10^{9}$ \\
\hline
\end{tabular}

